\title{
Étude de la diversité floristique par strates de quelques bois sacrés du Centre Bénin
}

\author{
Romaric Iralè EHINNOU KOUTCHIKA ${ }^{1 *}$, Daniel C. CHOUGOUROU2, Pierre O. AGBANI ${ }^{3}$, Brice \\ SINSIN11. \\ 1 Laboratoire d'Écologie Appliquée-Faculté des Sciences Agronomiques-Université d'Abomey-Calavi (Bénin) ; 01 BP \\ 526 Cotonou, Bénin ; Fax:00229 21303084 \\ 2. École Polytechnique d'Abomey-Calavi (EPAC) 01 BP 2009 Cotonou, Université d'Abomey-Calavi (UAC), Bénin. \\ 3. Centre Béninois de la Recherche Scientifique (CBRST/MESRS) 03BP1665 Cotonou, Bénin. Tél (+229) \\ 21321263/21320977, Fax : (+229) 21323671. \\ *Correspondance : E-mail : akofoudi2004@yahoo.fr; Cell : (+229) 900356 18/ (+229) 96080709 \\ Original submitted in on $25^{\text {th }}$ May 2013 Published online at www.m.elewa.org on 30 th September 2013. \\ https://dx.doi.org/10.4314/jab.v69i0.95068
}

\section{RÉSUMÉ}

Objectif : Cette étude vise à analyser la diversité floristique par strates de six bois sacrés au Centre Bénin.

Méthodologie et résultats : Cette diversité floristique par strates a été appréciée sur des placettes de 900 $\mathrm{m}^{2}$ de superficie suivant la méthode de Braun-Blanquet (1932) à partir de l'échantillonnage systématique. Sur ces placettes tous les ligneux de $d b h \geq 10$ sont mesurés, toutes les herbacées sont recensées et rangées. Dans la strate arborescente Ghézoun est plus diversifié, 16 espèces et 7 pour le moins diversifié, Winman. Au niveau de la strate arbustive, les bois sacrés Ghézoun et Winman sont plus diversifiés avec 14 espèces chacun et Agrazounvi faible, 6 espèces. La strate sous-arbustive est deux fois plus diversifiée (38 espèces) que les deux strates supérieures réunies. Le sous-bois est la plus riche en ressources floristiques de tous les bois sacrés.

Conclusion et application : L'étude met en évidence les potentialités floristiques par strate des bois sacrés ciblés. La strate sous arbustive est plus diversifiée que les deux autres (arborescente et arbustive). Mais, malgré qu'ils soient protégés par la population locale, quelques pressions sont perceptibles. A cet effet ces bois sacrés font appel à un programme d'aménagement en vue de leur développement durable.

Mots clés : Flore, strate, relevés phytosociologiques, transition, Centre Bénin

\begin{abstract}
Objectives: This study is an analysis of the floristic diversity in layers in six sacred woods in Central Benin. Methodology and results: This floristic diversity is appreciated on plots of $900 \mathrm{~m}^{2}$ following the method of Braun-Blanquet (1932) from systematic sampling. On these plots, all wood with a diameter at $1.3 \mathrm{~m}$ above ground or equal to $10 \mathrm{~cm}$ ( $\mathrm{dbh} \geq 10$ ) were measured. All herbs were identified and stored. "Ghézoun" in the tree stratum was more diversified with 16 species and "Winman" with 7 was the least diversified .At the shrub level, sacred woods "Ghézoun" and "Winman" were more diverse with 14 species each and "Agrazounvi" is low, with 6 species. Sub-shrub stratum is twice more diverse (38 species) than the two upper strata combined. The undergrowth is the richest in floristic resources of all sacred woods.
\end{abstract}


Conclusion and Application of results: The study highlights the floristic potential per stratum of the targeted sacred wood. The undergrowth is more diverse than the two other layers (tree and shrub). However, despite being protected by the local population, some pressures are noticeable. For this purpose, these sacred woods need an establishment of a development program for sustainable development.

Keywords : Flora, stratum, phytosociological plots, transition, Benin Centre

\section{INTRODUCTION}

Les bois sacrés constituent la forme endogène de conservation de la biodiversité en Afrique, en Asie et en Amérique latine (Swamy et al., 2003). Les bois sacrés sont constitués de bois, d'îlots de forêts, de savanes, de désert ou de steppes réservés aux cérémonies traditionnelles y compris les rituels magico-religieux (Essoh 2001 et 2003 ; Kaboré, 2010). Le bois sacré, est un îlot boisé au milieu d'une zone plus dégradée ou d'une zone cultivée (Alfiri, 2010). Les forêts sacrées (bois sacrés) sont des reliques de forêts à l'intérieur desquelles sont pratiqués des rites religieux (Garcia et al; 2006 ; Boukpéssi, 2010). La conservation des ressources biologiques est devenue une préoccupation majeure dans toutes les régions du monde. Face à la pression démographique, la flore et la faune sont sous menace permanente des activités anthropiques (Sokpon et Agbo, 2010). Au Centre Bénin, l'intensification des espaces agricoles n'épargne plus les bois sacrés qui jadis étaient considérés comme les sanctuaires de la biodiversité. Ces fragments forestiers étaient protégés par la législation traditionnelle (Agbo et Sokpon, 1998).

Aujourd'hui le constat est amer, le pouvoir des gestionnaires de ses reliques s'effrite de jour en jour face à l'avènement des religions révélées. Certains bois sacrés ont disparu et plusieurs autres ont vu leur superficie réduite. Ces espaces sacrés intéressent désormais les scientifiques et les organismes de protection de la nature. Certains chercheurs pensent que leur sauvegarde peut représenter une solution alternative pour la conservation de la diversité biologique (Kokou et al., 1999 ; Agbo et Sokpon, 1998; Kokou et Caballé 2000 ; Kokou et Sokpon, 2006; Kokou et Kokutse, 2006; Juhé-Beaulaton, 2010;
Hamberger, 2010; Kokou et Kokuster, 2010; Liberski- Bougnoud et al., 2010 ; Kaboré, 2010; Boukpessi, 2010). Malgré la petite superficie des bois sacrés dans leur majorité, ils se sont révélés comme un outil important de conservation de la biodiversité. lls constituent une sorte de pépinières et de réservoir génétique. La sacralisation de ces sites constituerait une nouvelle stratégie efficace à inclure dans les processus de gestion moderne de la biodiversité.

Malheureusement, le pouvoir des chefs traditionnels et des prêtres des divinités a baissé de sorte que depuis quelques années ces reliques forestières sont soumises à de forte exploitation. Aussi, la rareté des terres cultivables et la modernisation sans cesse croissante et irréversible mettent à mal les stratégies traditionnelles de conservation de la biodiversité par l'occupation horizontale des terres. Pour pallier à ce fait, le gouvernement béninois à traves la Direction Générale des Forêts et Ressources Naturelles (DGFRN) et le Programme des Nations Unies pour le Développement (PNUD) ont initié en 2011 le Programme d'Intégration des Forêts Sacrées dans le Système des Aires Protégées (PIFSAP) en vu de, l'aménagement et la conservation de la biodiversité des forêts sacrées. Actuellement, le diagnostic de conservation de la biodiversité par ces sites sacrés dresse un tableau relativement pessimiste face à l'intensification des activités agricoles dans le milieu d'étude (Oloukoi et al., 2006 ; Ehinnou Koutchika, 2009 ; Oloukoi, 2012).Voilà les raisons qui justifient l'intérêt de cette étude. L'objectif de ce travail est d'étudier la diversité floristique par strates de six bois sacrés au Centre Bénin.

\section{MATÉRIEL ET MÉTHODES}


Milieu d'étude : Le milieu d'étude est situé entre $2^{\circ}$ et $2^{\circ} 46^{\prime}$ de longitude Est et entre $7^{\circ} 30^{\prime}$ et $9^{\circ}$ de latitude

Nord. II couvre les Communes de Glazoué, de Savè et de Ouèssè (figure 1)

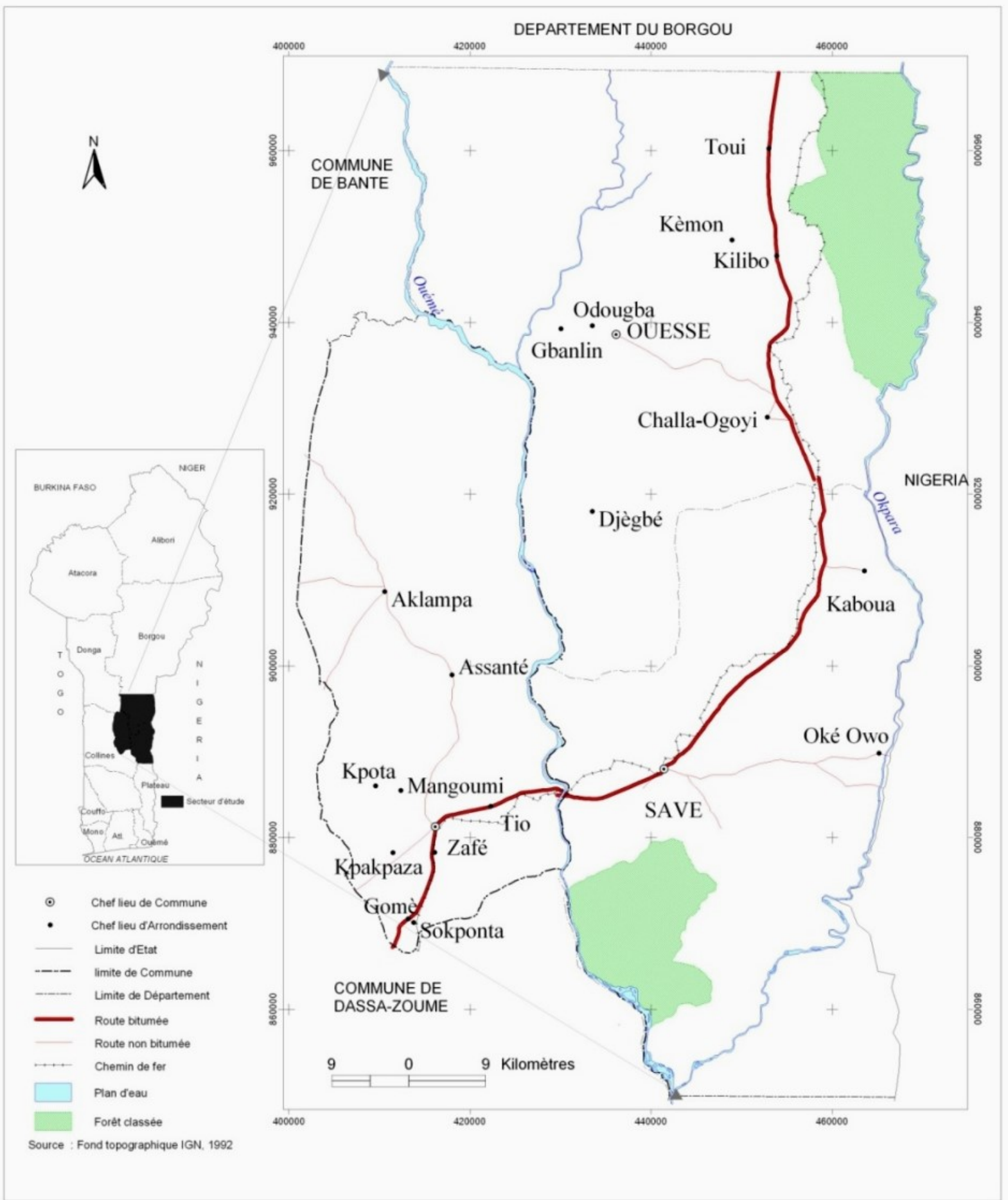

Figure 1 : Situation géographique du Centre Bénin

Le régime pluviométrique est bimodal avec une pluviométrie annuelle variant entre 900 et $1200 \mathrm{~mm} / \mathrm{an}$ d'eau répartie sur deux saisons. Les températures varient entre $22,5{ }^{\circ} \mathrm{C}$ et $33{ }^{\circ} \mathrm{C}$ avec une amplitude thermique moyenne de plus de $10{ }^{\circ} \mathrm{C}$. L'humidité relative varie entre $50,8 \%$ en janvier et $83,3 \%$ en août. 
Les valeurs minimales de l'humidité relative varient entre $27,5 \%$ en février et $65 \%$ en août. Le Centre Bénin est caractérisé par une pénéplaine dominée par des inselbergs. L'Ouémé, principal cours d'eau a un régime fluvial plus ou moins permanent. Ses débits varient entre 1020 et $1860 \mathrm{~m}^{3} / \mathrm{seconde}$ à la station de Savè (Oloukoi, 2012).

Méthodes: Les relevés phytosociologiques ont été effectués sur des placettes de $30 \mathrm{~m} \times 30 \mathrm{~m}$, soit $900 \mathrm{~m}^{2}$ de superficie, selon la méthode classique de BraunBlanquet (1932). Quarante cinq (45) relevés floristiques sont réalisés. Dans les bois sacrés de superficie $\leq 1 \mathrm{ha}$, les relevés sont distants de100 $\mathrm{m}$ et dans les autres bois sacrés de superficie $>1$ ha, les relevés sont installés à tous les $200 \mathrm{~m}$. Le diamètre à hauteur d'homme (dbh $\geq 10 \mathrm{~cm}$ ) à $1,30 \mathrm{~m}$ du sol ou à $30 \mathrm{~cm}$ au-dessus des contreforts des ligneux est mesuré à l'aide d'un ruban pi. Les coordonnées géographiques des placettes sont prises à l'aide d'un GPS (Global Positioning System). Pour ce travail, trois strates sont désignées par les lettres $A, B, C$.

$A$ : désigne la strate arborescente composée de ligneux de hauteur supérieure à $7 \mathrm{~m}$;

$B$ : désigne la strate arbustive constituée de ligneux de hauteur comprise entre 2 et $7 \mathrm{~m}$;

$C$ : désigne la strate sous-arbustive (sous-bois) composée des herbacées et des arbustes de hauteur inférieure à $2 \mathrm{~m}$.

\section{RÉSULTATS}

Diversité floristique : La diversité floristique par strate est appréciée dans les tableaux 1, 2, 3.

Diversité floristique de la strate arborescente : Dans la strate arborescente des six bois sacrés, Atchin et Ghézoun sont plus diversifiés et Winman, moins diversifié. Winman est le moins diversifié de la strate à
La richesse spécifique (No), est déterminée par le nombre total d'espèces de la surface observée ;

La diversité floristique a été appréciée en utilisant un certain nombre d'indices tels que :

L'Indice de diversité de Shannon ( $\left.\mathrm{H}^{\prime}\right)$, $H^{\prime}=-\sum P_{\text {ilog }} P_{i}$;

$P_{i}=n_{i} / N=$ nombre d'individus /espèces par strate ;

$\mathrm{N}=$ Nombre d'individus total /placette,

$H^{\prime}$ varie en général de 0 à 5 . Cet indice mesure la richesse spécifique des espèces par strate. II est utile car sa valeur augmente non seulement en fonction du nombre d'espèces, mais aussi selon l'abondance relative de chaque espèce dans la communauté. L'indice est maximal quand tous les individus sont répartis de façon égale sur toutes les espèces. Le coefficient de similitude de Sorensen est utilisé pour mesurer la ressemblance floristique entre les trois strates. Pour deux listes d'espèces $A$ et $B$, avec «a» comme nombre d'espèces de la strate arborescente, «b» comme nombre d'espèces de la strate arbustive et "C» comme nombre d'espèces communes aux deux strates, le coefficient de similitude de Sorensen (Ps) est calculé selon la formule suivante : $P s=2 c / a+b \times 100$. II varie de $0 \%$ à $100 \%$. Ps $=0$ quand il n'existe aucune espèce commune entre les deux strates. Ps atteint $100 \%$ quand les deux listes sont identiques.

cause de la dominance de Berlinia grandifolia au niveau de sa strate supérieure ; $H^{\prime}=1,91$ bits. Dans une forêt, quand l'indice de diversité de Shannon, ' $E$ $[0 ; 2,5]$, cela traduit la dominance d'une espèce ou d'un petit nombre d'espèces.

Tableau 1 : Récapitulatif du nombre( $\mathrm{Nb})$ de taxons, de la diversité floristique dans la strate arborescente des 6 bois sacrés étudiés

\begin{tabular}{lrrrrrr}
\hline & \multicolumn{1}{c}{ Atchin } & Agrazounvi & \multicolumn{1}{c}{ Djèzoun } & \multicolumn{1}{c}{ Ghézoun } & Monozoun & \multicolumn{2}{c}{ Winman } \\
\hline $\mathrm{Nb}$ Espèces & 7 & 10 & 12 & 15 & 12 & 13 \\
$\mathrm{Nb}$ Genres & 7 & 10 & 12 & 13 & 12 & 13 \\
$\mathrm{Nb}$ Familles & 6 & 8 & 7 & 8 & 7 & 10 \\
Richesse spécifique & 15 & 10 & 12 & 16 & 12 & 7 \\
Indice de Shannon $\left(\mathrm{H}^{\prime}\right)$ & 3,33 & 2,31 & 2,29 & 4,5 & 3,12 & 1,91 \\
\hline
\end{tabular}

\section{Diversité floristique de la strate arbustive}


Tableau 2: Récapitulatif du nombre(Nb) de taxons, de la diversité floristique dans la strate arbustive des 6 bois sacrés étudiés

\begin{tabular}{lllllll}
\hline & Atchin & Agrazounvi & Djèzoun & Ghézoun & Monozoun & Winman \\
\hline $\mathrm{Nb}$ Espèces & 14 & 6 & 11 & 14 & 12 & 7 \\
$\mathrm{Nb}$ Genres & 13 & 6 & 11 & 12 & 11 & 7 \\
$\mathrm{Nb}$ Familles & 11 & 6 & 8 & 7 & 10 & 6 \\
Richesse spécifique & 7 & 6 & 11 & 14 & 12 & 14 \\
Indice de Shannon $\left(\mathrm{H}^{\prime}\right)$ & 1,94 & 2,16 & 2,74 & 3,43 & 3,5 & 3,5 \\
\hline
\end{tabular}

Dans la strate arbustive, des six bois sacrés, quatre sont plus diversifiés (Djèzoun, Ghézoun, Monozoun et Winman). Les bois sacrés Agrazounvi et Atchin sont peu diversifiés. L'indice de diversité de Shannon $\left(\mathrm{H}^{\prime}\right)$ est de 3,29 en moyenne dans les bois sacrés plus diversifiés. Cet indice $\left(H^{\prime}\right) \in[2,6 ; 3,9]$, la strate arbustive est supposée moyenne. Ces reliques forestières ont bénéficié une protection de la part des populations locales.

Tableau 3: Récapitulatif du nombre( $\mathrm{Nb})$ de taxons, de la diversité floristique dans le sous-bois des 6 bois sacrés étudiés

\begin{tabular}{lllllll}
\hline & Atchin & Agrazounvi & Djèzoun & Ghézoun & Monozoun & Winman \\
\hline Nb Espèces & 19 & 28 & 29 & 16 & 23 & 27 \\
Nb Genres & 16 & 28 & 28 & 16 & 23 & 27 \\
Nb Familles & 15 & 21 & 19 & 13 & 18 & 21 \\
Richesse spécifique & 28 & 35 & 38 & 17 & 15 & 20 \\
Indice de Shannon (H') & 4,69 & 4,55 & 4,78 & 3,99 & 4,55 & 4,32 \\
\hline
\end{tabular}

Le sous-bois des six (6) bois sacrés se révèle deux fois diversifiés que chacune des deux strates supérieures. Dans ces bois sacrés, cinq sont très diversifiés (Atchin, Agrazounvi, Djèzoun, Monozoun et Winman). Ces reliques ont leurs indices de diversité de Shannon $H^{\prime} \in[4 ; 6[$; ce qui montre que les espèces tendent vers l'équiprobalité à cause de l'abondance des herbacées. Cet apport est justifié par l'abondance de l'élevage, de la faune aviaire par zoochorie et de la transhumance dans le milieu d'étude. L'indice de Shannon $\left(H^{\prime}\right)$ calculé pour l'ensemble des six (6) bois sacrés par strate donne une moyenne de 2,9 pour la strate arborescente, 2,8 pour la strate arbustive et 4,47 pour la strate sous-arbustive. En considérant séparément les trois strates, les indices de Shannon $\left(H^{\prime}\right)$ calculés, sont compris entre 1,9 et 4,5 pour la strate arborescente, entre 1,9 et 3,5 pour la strate arbustive et entre 3,9 et 4,7 pour la strate sousarbustive. Le sous-bois est pourvu de l'indice de diversité le plus élevé à cause des herbacées et de la densité de régénération des espèces végétales. L'indice de Shannon dans les strates arborescente et arbustive est faible. Dans les strates arborescente et arbustive, $H^{\prime} \in[2,6 ; 3,9]$, la strate est supposée moyenne. Leur diversité floristique est moins nette. Dans la strate arbustive, on note la présence de certaines espèces en transition comme: Ceiba penatandra, Milicia excelsa, Andansonia digitata, Cola gigantea, Berlinia grandifolia, Vitex doniana, Lannea acida, Lannea kerstingui, Anogeisus leiocarpa etc. qui, dans leur jeune âge appartiennent à la strate arbustive au lieu de la strate arborescente plus tard. Cette strate est le siège des espèces régénérées et des herbacées. Elle héberge la plupart des espèces appartenant aux autres strates.

Ressemblance floristique par strate: Le coefficient de Sorensen est utilisé dans le tableau 4 pour mesurer la ressemblance floristique dans les trois strates.

Tableau 4 : Coefficients de similarité entre les trois strates

\begin{tabular}{llll}
\hline Strates & Nombre d'espèces & Espèces communes & Coefficient de similarité Ps \\
\hline Strate arborescente & 39 & 23 & 59,7
\end{tabular}


Koutchika et al. J. Appl. Biosci. 2013. Étude de la diversité floristique par strates de quelques bois sacrées du Centre Bénin

\begin{tabular}{llll}
\hline Strate arbustive & 38 & & \\
\cline { 1 - 2 } Strate arborescente & 39 & 13 & 22,8 \\
\cline { 1 - 2 } Strate sous-arbustive & 75 & 17 & 30 \\
\hline Strate arbustive & 38 & 17 & \\
\hline Strate sous-arbustive & 75 & &
\end{tabular}

Une comparaison deux à deux de la composition floristique des trois strates, par la méthode de Sorensen, indique un seuil de similitude compris entre 22 et $59 \%$ (Tableau 5). Elle montre que les différentes strates étudiées ne présenteraient pas la même diversité floristique.

Diversité floristique par bois sacré : Le tableau 5 présente la diversité floristique par bois sacré.

Des six bois sacrés, l'indice de Shannon est 4,5 pour Ghézoun. Ghézoun est plus diversifié dans ses deux strates supérieures (arborescente et arbustive) que les autres bois sacrés sauf Winman ou H' $=3,5$ dans la strate arbustive. Ghézoun est très protégé par la population locale. La diversité spécifique dans la strate arborescente est très faible dans Winman, à cause de la dominance de Berlinia grandifolia. Le sous-bois de Agrazounvi est peu diversifié à cause du forte peuplement de Clausena anisata. Dans le sous-bois, l'indice de Shannon est 4,75 dans Djèzoun, il est le plus diversifié. Monozoun est sous forte pression anthropique et le bois sacré Agrazounvi est en reconstitution.

Tableau 5 : Comparaison de la diversité floristique par strate et par bois sacré

\begin{tabular}{|c|c|c|c|c|c|c|}
\hline Paramètres & Atchin & Agrazounvi & Djèzoun & Ghézoun & Monozoun & Winman \\
\hline \multicolumn{7}{|l|}{ Strate arborescente } \\
\hline Richesse spécifique & 15 & 10 & 12 & 16 & 12 & 7 \\
\hline $\begin{array}{l}\text { Indice de Shannon (H') } \\
\text { Strate arbustive }\end{array}$ & 3,33 & 2,31 & 2,29 & 4,5 & 3,12 & 1,91 \\
\hline Richesse spécifique & 7 & 6 & 11 & 14 & 12 & 14 \\
\hline $\begin{array}{l}\text { Indice de Shannon (H') } \\
\text { Strate sous-arbustive }\end{array}$ & 1,94 & 2,16 & 2,74 & 3,43 & 3,5 & 3,5 \\
\hline Richesse spécifique & 28 & 35 & 38 & 17 & 15 & 20 \\
\hline Indice de Shannon $\left(\mathrm{H}^{\prime}\right)$ & 4,69 & 4,55 & 4,78 & 3,99 & 4,5 & 4,32 \\
\hline
\end{tabular}

\section{DISCUSSION}

La diversité floristique varie d'une strate à une autre. Le sous-bois est la strate la plus diversifiée. Les espèces recensées par strate montrent que la strate la plus diversifiée est celle utilisée par les populations pour leurs besoins quotidiens (la recherche des plantes médicinales, les tiges et les racines de certaines espèces sont récoltées et vendues sur le marché local et utilisées comme brosse végétale, le tronc de certains arbres sert à tirer des traverses pour la toiture des maisons etc.). II est suivi de la strate arbustive, la plus ou moins protégée. Bien que les populations locales aient des pratiques dans cette strate, elle a conservé une certaine richesse spécifique. Ce qui est confirmé par l'indice de diversité de Shannon très élevé. Les strates dans lesquelles les agriculteurs mènent des activités, ont un indice de Shannon légèrement supérieur à la moyenne de l'ensemble des relevés $(\mathrm{H}$ $=3,5$ ). La strate sous-arbustive est la plus riche en flore. La diversité floristique est faible dans les strates supérieures. Environ 90\% de leur flore se trouvent dans les strates inférieures (arbustive et sous-arbustive). Le sous-bois a un recouvrement de plus de $90 \%$ dans les bois sacrés dominés par Chromolena odorata (Ashampong), Andropogon gayanus (l'Andropogon) et Anchomanes welwitschii (Parapluie de singe). La forte dominance des herbacées dans certains bois sacrés montre qu'ils sont sous pression anthropique. L'homme, les troupeaux et le feu entretiennent ces perturbations. Les grands arbres fréquemment rencontrés dans la strate arborescente sont: Milicia excelsa (Iroko), Antiaris toxicaria (Faux iroko), Ceiba pentandra (Fromager), Berlinia grandifolia (le Berlinia), Andansonia digitata (Baobab), Cola gigantea (le Cola), Vitex doniana (Prunier noir), Lannea acida (les lannea), Lannea kerstingui (les lannea) et Anogeisus leiocarpa (Bouleau d'Afrique). Dans le parc national de la Marahoué, Centre-Ouest de la Côte d'Ivoire, l'indice de diversité de Shannon et l'équitabilité de la forêt 1 sont 
respectivement de 7,805 bits et de 0,972. L'indice de diversité de Shannon et l'équitabilité de la forêt 2 sont respectivement de 6,943 bits et de 0,975. Au niveau de la forêt 3, l'indice de diversité de Shannon et l'équitabilité sont respectivement de 7,154 bits et de 0,965 . Cela permet de montrer que le foret 1 est plus diversifié que le foret 3 . La variation des indices de diversité est en rapport avec le nombre d'espèces. Dans le sous-bois des plantations de teck étudié par Djego (2006), les valeurs de l'indice de diversité de Shannon varient de 4,6 à 4,9 bits dans les forêts naturelles et de 1,2 à 3,9 bits dans le sous-bois dans les plantations d'essences exotiques. L'équitabilité de

\section{CONCLUSION}

Les inventaires réalisés ont permis de recenser un grand nombre d'espèces. Bien que sous pressions humaines permanentes, les bois sacrés du Centre Bénin conservent encore leur fond floristique. La qualité de certaines espèces de ce cortège, les espèces menacées de la liste rouge de l'Union Internationale pour la Conservation de la Nature (UICN) comme: Afzelia africana (Haricot acajou), Borassus aethiopum (Rônier), Khaya senegalensis (Cailcédrat), Milicia excelsa (Iroko), Ptereocarpus erinaceus (Veine) Vitellaria paradoxa (Karité) et Zanthoxylum

\section{RÉFÉRENCES BIBLIOGRAPHIQUES}

Agbo V. Sokpon N. 1998. Forêts sacrées et patrimoine vital au Bénin. Rapport Technique final du Projet CRDI n 95-8170. Université National du Bénin, Faculté des Sciences Agronomiques, $104 \mathrm{p}$.

Alfieri C. 2010. Rempart végétal et bois sacrés, dans " Forêts sacrées et sanctuaires boisées, Des créations culturelles et botaniques (Burkina Faso, Togo, Bénin » pp. 185-199

Boukpessi T. 2010. Les pratiques endogènes de conservation de la biodiversité au CentreTogo. Université de Lomé et de franchecomité, thèse de doctorat unique en Géographie, 206 230.

Braun-Blanquet J. 1932. Plant sociology the study of plan communities.Transfland revised and edited by FULLERG D. \& Conard H.S., 356390.

Garcia CJ, Pascal P, Kushalappa GG. 2006. Les forêts sacrées du Kadagu en Inde: écologie et religion. Bois et Forêts des Tropiques 288, 513.
Pielou est plus élevée $(0,88$ à 0,95$)$ dans le sous-bois et plus faible $(0,26$ à 0,8$)$ par rapport à celui des plantations d'essences exotiques. Ces deux paramètres sont utilisés pour mieux apprécier la diversité spécifique du sous-bois. L'indice de diversité de Shannon relativement faible dans certains bois sacrés pourrait être imputé aux activités de mise en culture qui se sont déroulées dans certains bois sacrés il ya quelques années. Aussi Szawagrzyk et Gazda (2007) montrent-ils que la grande diversité spécifique conduit à la stabilité et à la forte productivité des écosystèmes de savane.

zanthozyloides (le Fagara) sont encore perceptibles dans ces reliques forestières. Cela montre l'importance des bois sacrés dans la conservation des espèces menacées de disparition. Cette étude montre aussi que, lorsque les activités de conservation se limitent aux seuls gestionnaires des bois sacrés, beaucoup de choses restent à faire pour préserver ces endroits sacrés. Dans la structure de la végétation, des différences apparaissent car les grands arbres sont parfois coupés dans certains bois sacrés.

Ehinnou Koutchika I R, 2009. État et interactions des modes d'occupation des terres (cotontranshumance - bois sacrés) dans la Commune de Glazoué, Mémoire de DEA, École doctorale pluridisciplinaire de I'Université d'Abomey-Calavi 82p

Essoh E. 2001. Repenser le concept de développement durable-in cahiers du GRATICE-Université de Paris XII-pp. 135-147

Essoh E. 2003. Plaider pour un patrimoine des bois sacrés en Afrique Noire, Actes du Xlle Congrès Forestier Mondial-Québec Canada "La forêt, source de vie » Tome A « des forêts pour les gens » - FAO, 381p.

Hamberger K. 2010. Perspectives de la brousse, la fonction symbolique des forêts sacrées en pays Ouachi (Sud-est Togo), in Forêts sacrées et sanctuaires boisés des créations culturelles et biologiques

Jué-Beaulaton D.2010. Forêts sacrées et sanctuaires boisées, Des créations culturelles et botaniques (Burkina Faso, Togo, Bénin), 207 232. 
Kaboré A. 2010. Les stratégies communautaires d'adaptation au changement climatique: Cas des bois sacrés dans l'aire socioculturelle Moaaga du Burkina Faso. Thèse de doctorat unique en géographie de l'Université d'Abomey-Calavi au Bénin, 187- 2014.

Kokou K, Caballé G, Akpagana K, 1999. Analyse floristique des îlots forestiers du sud du Togo. Acta Botanica Gallica 146 (2: 139-144).

Kokou K. Caballé G., 2000. Les îlots forestiers de la plaine côtière togolaise. Bois et Forêts des Tropiques, $n^{\circ} 263$ (1) pp. 39-51.

Kokou K, Adjossou K, Hamberger K. 2005. Les forêts sacrées de l'aire ouatchi au sud-est du Togo et les contraintes actuelles des modes de gestion locale des ressources forestières, VertigO 6, 3./vertigo.revues.org/8661; DOI: 10.4000/vertigo.866.

Kokou K, Sokpon N. 2006. Les Forêts sacrées du couloir du Dahomey. Bois et forêts des tropiques $n^{\circ} 288$ (2), 15-23.

Kokou k. Kokutse A. D., 2006. Rôle de la régénération naturelle dans la dynamique actuelle des forêts sacrées littorales du Togo. Phytocoenologia, 36 (2) : 403-419.

Kokou K. Sokpon N., 2006. Les Forêts sacrées du couloir du Dahomey. Bois et forêts des tropiques $n^{\circ} 288$ (2), pp.15-23.

Kokou K. Kokuster A. 2010. Des forêts sacrées, dans la région du littorale très anthropisée du sud Togo, dans Forêts sacrées et sanctuaires boisés des créations culturelles et biologiques (Burkina-Faso, Togo, Bénin Edition Karthala, $91-122$.

Liberski- Bougnoud D, Fourie A, Nigna S. 2010. Les "bois sacrés" faits et illusions à propos des santuaires boisés des Kasena (Burkina Faso, in Forêts sacrées et sanctuaires boisés des créations culturelles et biologiques (BurkinaFaso, Togo, Bénin Edition Karthala, 59 -90.

Oloukoi J, MAMA VJ, AGBO FB, 2006. Modélisation de la dynamique de l'occupation des terres dans le Département des collines au Bénin, Télédétection, 2006, vol. 6, $n^{\circ} 4$, pp. 305-323

Oloukoi J. 2012. Utilité de la télédétection et des systèmes d'information géographique dans l'étude de la dynamique spatiale de l'occupation des terres au Centre Bénin. 306p.

Sokpon N. \& Agbo V. 2010. Forêts sacrées et patrimoine au Bénin in Atlas de la biodiversité de l'Afrique de l'ouest, Tome 1 pp 536-547.
Swamy P.S., 2003. Spirituality and ecology of sacred groves in Tamil Nadu, India. Unasylva 213, vol 54, pp.53-58

Szwagrzyk J. \& Gazda A. 2007. Above-ground standing biomass and tree species diversity in natural stands of Central Europe. Journal of vegetation Science, 18: 555-562, 